\title{
Asymptotic Behavior of the Solutions of System of Difference Equations of Exponential Form
}

\author{
Vu Van Khuong ${ }^{1,2}$ and Tran Hong Thai ${ }^{1}$ \\ ${ }^{1}$ Department of Mathematics, Hung Yen University of Technology and Education, Khoai Chau, Hung Yen 393008, Vietnam \\ ${ }^{2}$ Department of Mathematical Analysis, University of Transport and Communications, Dong Da, Hanoi 10200, Vietnam
}

Correspondence should be addressed to Vu Van Khuong; vuvankhuong@gmail.com

Received 31 May 2014; Revised 10 September 2014; Accepted 24 September 2014; Published 13 October 2014

Academic Editor: Honglei Xu

Copyright (c) 2014 V. Van Khuong and T. Hong Thai. This is an open access article distributed under the Creative Commons Attribution License, which permits unrestricted use, distribution, and reproduction in any medium, provided the original work is properly cited.

\begin{abstract}
The goal of this paper is to study the boundedness, the persistence, and the asymptotic behavior of the positive solutions of the system of two difference equations of exponential form: $x_{n+1}=\left(a+b e^{-y_{n}}+c e^{-x_{n}}\right) /\left(d+h y_{n}\right), y_{n+1}=\left(a+b e^{-x_{n}}+c e^{-y_{n}}\right) /\left(d+h x_{n}\right)$, where $a, b, c, d$, and $h$ are positive constants and the initial values $x_{0}, y_{0}$ are positive real values. Also, we determine the rate of convergence of a solution that converges to the equilibrium $E=(\bar{x}, \bar{y})$ of this system.
\end{abstract}

\section{Introduction}

In [1], the authors studied the boundedness, the asymptotic behavior, the periodicity, and the stability of the positive solutions of the difference equation:

$$
y_{n+1}=\frac{\alpha+\beta e^{-y_{n}}}{\gamma+y_{n-1}},
$$

where $\alpha, \beta, \gamma$ are positive constants and the initial values $y_{-1}, y_{0}$ are positive numbers.

Motivated by the above paper we will extend the above difference equation to a system of difference equations; our goal will be to investigate the boundedness, the persistence, and the asymptotic behavior of the positive solutions of the following system of exponential form:

$$
\begin{aligned}
& x_{n+1}=\frac{a+b e^{-y_{n}}+c e^{-x_{n}}}{d+h y_{n}}, \\
& y_{n+1}=\frac{a+b e^{-x_{n}}+c e^{-y_{n}}}{d+h x_{n}},
\end{aligned}
$$

where $a, b, c, d, h$ are positive constants and the initial values $x_{0}, y_{0}$ are positive real values.
Difference equations and systems of difference equations of exponential form can be found in [2-6]. Moreover, as difference equations have many applications in applied sciences, there are many papers and books that can be found concerning the theory and applications of difference equations; see [7-9] and the references cited therein.

\section{Global Behavior of Solutions of System (2)}

In the first lemma we study the boundedness and persistence of the positive solutions of (2).

Lemma 1. Every positive solution of (2) is bounded and persists.

Proof. Let $\left(x_{n}, y_{n}\right)$ be an arbitrary solution of (2). From (2) we can see that

$$
x_{n} \leq \frac{a+b+c}{d}, \quad y_{n} \leq \frac{a+b+c}{d}, \quad n=1,2, \ldots
$$


In addition, from (2) and (3) we get

$$
\begin{array}{r}
x_{n} \geq \frac{a+b e^{-(a+b+c) / d}+c e^{-(a+b+c) / d}}{d+h \cdot((a+b+c) / d)}, \\
y_{n} \geq \frac{a+b e^{-(a+b+c) / d}+c e^{-(a+b+c) / d}}{d+h \cdot((a+b+c) / d)}, \\
n=2,3, \ldots .
\end{array}
$$

Therefore, from (3) and (4) the proof of the lemma is complete.

In order to prove the main result of this section, we recall the next theorem without its proof. See $[10,11]$.

Theorem 2. Let $\mathscr{R}=\left[a_{1}, b_{1}\right] \times\left[c_{1}, d_{1}\right]$ and

$$
f: \mathscr{R} \longrightarrow\left[a_{1}, b_{1}\right], \quad g: \mathscr{R} \longrightarrow\left[c_{1}, d_{1}\right]
$$

be a continuous functions such that the following hold:

(a) $f(x, y)$ is decreasing in both variables and $g(x, y)$ is decreasing in both variables for each $(x, y) \in \mathscr{R}$;

(b) if $\left(m_{1}, M_{1}, m_{2}, M_{2}\right) \in \mathscr{R}^{2}$ is a solution of

$$
\begin{array}{ll}
M_{1}=f\left(m_{1}, m_{2}\right), & m_{1}=f\left(M_{1}, M_{2}\right), \\
M_{2}=g\left(m_{1}, m_{2}\right), & m_{2}=g\left(M_{1}, M_{2}\right)
\end{array}
$$

then $m_{1}=M_{1}$ and $m_{2}=M_{2}$. Then the following system of difference equations,

$$
x_{n+1}=f\left(x_{n}, y_{n}\right), \quad y_{n+1}=g\left(x_{n}, y_{n}\right),
$$

has a unique equilibrium $(\bar{x}, \bar{y})$ and every solution $\left(x_{n}, y_{n}\right)$ of the system (7) with $\left(x_{0}, y_{0}\right) \in \mathscr{R}$ converges to the unique equilibrium $(\bar{x}, \bar{y})$. In addition, the equilibrium $(\bar{x}, \bar{y})$ is globally asymptotically stable.

Now we state the main theorem of this section.

Theorem 3. Consider system (2). Suppose that the following relation holds true:

$$
b+c<d
$$

Then system (2) has a unique positive equilibrium $(\bar{x}, \bar{y})$ and every positive solution of (2) tends to the unique positive equilibrium $(\bar{x}, \bar{y})$ as $n \rightarrow \infty$. In addition, the equilibrium $(\bar{x}, \bar{y})$ is globally asymptotically stable.

Proof. We consider the functions

$$
\begin{aligned}
& f(u, v)=\frac{a+b e^{-v}+c e^{-u}}{d+h v} \\
& g(u, v)=\frac{a+b e^{-u}+c e^{-v}}{d+h u}
\end{aligned}
$$

where

$$
u, v \in I=\left[\frac{a+(b+c) e^{-(a+b+c) / d}}{c+h \cdot((a+b+c) / d)}, \frac{a+b+c}{d}\right] .
$$

It is easy to see that $f(u, v), g(u, v)$ are decreasing in both variables for each $(u, v) \in I \times I$. In addition, from (9) and (10) we have $f(u, v) \in I, g(u, v) \in I$ as $(u, v) \in I \times I$ and so $f: I \times I \rightarrow I, g: I \times I \rightarrow I$.

Now let $m_{1}, M_{1}, m_{2}, M_{2}$ be positive real numbers such that

$$
\begin{aligned}
& M_{1}=\frac{a+b e^{-m_{2}}+c e^{-m_{1}}}{d+h m_{2}}, \\
& M_{2}=\frac{a+b e^{-m_{1}}+c e^{-m_{2}}}{d+h m_{1}}, \\
& m_{1}=\frac{a+b e^{-M_{2}}+c e^{-M_{1}}}{d+h M_{2}}, \\
& m_{2}=\frac{a+b e^{-M_{1}}+c e^{-M_{2}}}{d+h M_{1}} .
\end{aligned}
$$

Moreover arguing as in the proof of Theorem 2, it suffices to assume that

$$
m_{1} \leq M_{1}, \quad m_{2} \leq M_{2}
$$

From (11) we get

$$
\begin{aligned}
& b e^{-m_{2}}+c e^{-m_{1}}=\left(d+h m_{2}\right) M_{1}-a, \\
& b e^{-M_{2}}+c e^{-M_{1}}=\left(d+h M_{2}\right) m_{1}-a, \\
& b e^{-m_{1}}+c e^{-m_{2}}=\left(d+h m_{1}\right) M_{2}-a, \\
& b e^{-M_{1}}+c e^{-M_{2}}=\left(d+h M_{1}\right) m_{2}-a,
\end{aligned}
$$

which imply that

$$
\begin{aligned}
d\left(M_{1}\right. & \left.-m_{1}\right)+h\left(M_{1} m_{2}-M_{2} m_{1}\right) \\
& =b\left(e^{-m_{2}}-e^{-M_{2}}\right)+c\left(e^{-m_{1}}-e^{-M_{1}}\right) \\
& =b e^{-m_{2}-M_{2}}\left(e^{M_{2}}-e^{m_{2}}\right)+c e^{-m_{1}-M_{1}}\left(e^{M_{1}}-e^{m_{1}}\right), \\
d\left(M_{2}-m_{2}\right)+h\left(M_{2} m_{1}-M_{2} m_{2}\right) & \\
= & b\left(e^{-m_{1}}-e^{-M_{1}}\right)+c\left(e^{-m_{2}}-e^{-M_{2}}\right) \\
& =b e^{-m_{1}-M_{1}}\left(e^{M_{1}}-e^{m_{1}}\right)+c e^{-m_{2}-M_{2}}\left(e^{M_{2}}-e^{m_{2}}\right) .
\end{aligned}
$$

Moreover, we get

$$
\begin{array}{ll}
e^{M_{1}}-e^{m_{1}}=e^{\alpha}\left(M_{1}-m_{1}\right), & m_{1} \leq \alpha \leq M_{1}, \\
e^{M_{2}}-e^{m_{2}}=e^{\beta}\left(M_{2}-m_{2}\right), & m_{2} \leq \beta \leq M_{2} .
\end{array}
$$

Then by adding the two relations (14) we obtain

$$
\begin{aligned}
d( & \left.M_{1}-m_{1}\right)+d\left(M_{2}-m_{2}\right) \\
= & (b+c) \\
\quad & {\left[e^{-m_{1}-M_{1}+\alpha}\left(M_{1}-m_{1}\right)+e^{-m_{2}-M_{2}+\beta}\left(M_{2}-m_{2}\right)\right] . }
\end{aligned}
$$


Therefore from (16) we have

$$
\begin{aligned}
& \left(M_{1}-m_{1}\right)\left[d-(b+c) e^{-m_{1}-M_{1}+\alpha}\right] \\
& \quad+\left(M_{2}-m_{2}\right)\left[d-(b+c) e^{-m_{2}-M_{2}+\beta}\right]=0 .
\end{aligned}
$$

Then using (8), (12), and (17) gives us $m_{1}=M_{1}$ and $m_{2}=$ $M_{2}$. Hence from Theorem 2 system (2) has a unique positive equilibrium $(\bar{x}, \bar{y})$ and every positive solution of (2) tends to the unique positive equilibrium $(\bar{x}, \bar{y})$ as $n \rightarrow \infty$. In addition, the equilibrium $(\bar{x}, \bar{y})$ is globally asymptotically stable. This completes the proof of the theorem.

\section{Rate of Convergence}

In this section we give the rate of convergence of a solution that converges to the equilibrium $E=(\bar{x}, \bar{y})$ of the system (2) for all values of parameters. The rate of convergence of solutions that converge to an equilibrium has been obtained for some two-dimensional systems in $[12,13]$.

The following results give the rate of convergence of solutions of a system of difference equations:

$$
\mathbf{x}_{n+1}=[A+B(n)] \mathbf{x}_{n}
$$

where $\mathbf{x}_{n}$ is a $k$-dimensional vector, $A \in \mathbf{C}^{k \times k}$ is a constant matrix, and $B: \mathbb{Z}^{+} \rightarrow C^{k \times k}$ is a matrix function satisfying

$$
\|B(n)\| \longrightarrow 0 \quad \text { when } n \longrightarrow \infty
$$

where $\|\cdot\|$ denotes any matrix norm which is associated with the vector norm; $\|\cdot\|$ also denotes the Euclidean norm in $\mathbb{R}^{2}$ given by

$$
\|\mathbf{x}\|=\|(x, y)\|=\sqrt{x^{2}+y^{2}} .
$$

$$
J_{T}=\left(\begin{array}{c}
\frac{-c e^{-x}}{d+h y} \\
\frac{-b e^{-x}(d+h x)-h \cdot\left(a+b e^{-x}+c e^{-y}\right)}{(d+h x)^{2}}
\end{array}\right.
$$

By using the system (23), value of the Jacobian matrix of $T$ at the equilibrium point $E=(\bar{x}, \bar{y})=(\bar{x}, \bar{x})$ is
Theorem 4 (see [14]). Assume that condition (19) holds. If $\mathbf{x}_{n}$ is a solution of system (18), then either $\mathbf{x}_{n}=0$ for all large $n$ or

$$
\rho=\lim _{n \rightarrow \infty} \sqrt[n]{\left\|\mathbf{x}_{n}\right\|}
$$

exists and is equal to the modulus of one of the eigenvalues of matrix A.

Theorem 5 (see [14]). Assume that condition (19) holds. If $\mathbf{x}_{n}$ is a solution of system (18), then either $\mathbf{x}_{n}=0$ for all large $n$ or

$$
\rho=\lim _{n \rightarrow \infty} \frac{\left\|\mathbf{x}_{n+1}\right\|}{\left\|\mathbf{x}_{n}\right\|}
$$

exists and is equal to the modulus of one of the eigenvalues of matrix A.

The equilibrium point of the system (2) satisfies the following system of equations:

$$
\begin{aligned}
& \bar{x}=\frac{a+b e^{-\bar{y}}+c e^{-\bar{x}}}{d+h \cdot \bar{y}}, \\
& \bar{y}=\frac{a+b e^{-\bar{x}}+c e^{-\bar{y}}}{d+h \cdot \bar{x}} .
\end{aligned}
$$

If $b+c<d$, we can easily see that the system (23) has an unique equilibrium $E=(\bar{x}, \bar{x})$.

The map $T$ associated with the system (2) is

$$
T(x, y)=\left(\begin{array}{l}
f(x, y) \\
g(x, y)
\end{array}\right)=\left(\begin{array}{c}
\frac{a+b e^{-y}+c e^{-x}}{d+h y} \\
\frac{a+b e^{-x}+c e^{-y}}{d+h x}
\end{array}\right) .
$$

The Jacobian matrix of $T$ is

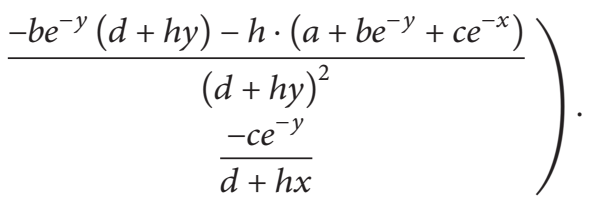

$$
J_{T}=\left(\begin{array}{cc}
\frac{-c e^{-\bar{x}}}{d+h \bar{x}} & \frac{-b e^{-\bar{x}}(d+h \bar{x})-h \cdot\left[a+(b+c) e^{-\bar{x}}\right]}{(d+h \bar{x})^{2}} \\
\frac{-b e^{-\bar{x}}(d+h \bar{x})-h \cdot\left[a+(b+c) e^{-\bar{x}}\right]}{(d+h \bar{x})^{2}} & \frac{-c e^{-\bar{x}}}{d+h \bar{x}}
\end{array}\right) .
$$


Our goal in this section is to determine the rate of convergence of every solution of the system (2) in the regions where the parameters $a, b, c, d, h \in(0, \infty),(b+c<d)$ and initial conditions $x_{0}$ and $y_{0}$ are arbitrary, nonnegative numbers.

Theorem 6. The error vector $\mathbf{e}_{n}=\left(\begin{array}{c}e_{n}^{1} \\ e_{n}^{2}\end{array}\right)=\left(\begin{array}{l}x_{n}-\bar{x} \\ y_{n}-\bar{y}\end{array}\right)$ of every solution $\mathbf{x}_{n} \neq \mathbf{0}$ of (2) satisfies both of the following asymptotic relations:

$$
\begin{aligned}
& \lim _{n \rightarrow \infty} \sqrt[n]{\left\|\mathbf{e}_{n}\right\|}=\left|\lambda_{i}\left(J_{T}(E)\right)\right| \quad \text { for some } i=1,2, \\
& \lim _{n \rightarrow \infty} \frac{\left\|\mathbf{e}_{n+1}\right\|}{\left\|\mathbf{e}_{n}\right\|}=\left|\lambda_{i}\left(J_{T}(E)\right)\right| \quad \text { for some } i=1,2,
\end{aligned}
$$

where $\left|\lambda_{i}\left(J_{T}(E)\right)\right|$ is equal to the modulus of one of the eigenvalues of the Jacobian matrix evaluated at the equilibrium $J_{T}(E)$.

Proof. First, we will find a system satisfied by the error terms. The error terms are given as

$$
\begin{aligned}
& x_{n+1}-\bar{x}=\frac{a+b e^{-y_{n}}+c e^{-x_{n}}}{d+h y_{n}}-\frac{a+b e^{-\bar{y}}+c e^{-\bar{x}}}{d+h \bar{y}} \\
& =\left(\left(a+b e^{-y_{n}}+c e^{-x_{n}}\right)(d+h \bar{y})\right. \\
& \left.-\left(a+b e^{-\bar{y}}+c e^{-\bar{x}}\right)\left(d+h y_{n}\right)\right) \\
& \times\left(\left(d+h y_{n}\right)(d+h \bar{y})\right)^{-1} \\
& =\left(b d\left(e^{-y_{n}}-e^{-\bar{y}}\right)+c d\left(e^{-x_{n}}-e^{-\bar{x}}\right)\right. \\
& +a h\left(\bar{y}-y_{n}\right)+b h\left(e^{-y_{n}} \bar{y}-e^{-\bar{y}} y_{n}\right) \\
& \left.+\operatorname{ch}\left(e^{-x_{n}} \bar{y}-e^{-\bar{x}} y_{n}\right)\right) \\
& \times\left(\left(d+h y_{n}\right)(d+h \bar{y})\right)^{-1} \\
& =\frac{-b d\left(e^{y_{n}}-e^{\bar{y}}\right)}{e^{y_{n}+\bar{y}}\left(d+h y_{n}\right)(d+h \bar{y})} \\
& +\frac{-c d\left(e^{x_{n}}-e^{\bar{x}}\right)}{e^{x_{n}+\bar{x}}\left(d+h y_{n}\right)(d+h \bar{y})} \\
& +\frac{b h}{\left(d+h y_{n}\right)(d+h \bar{y})} \\
& \times\left(e^{-y_{n}} \bar{y}-e^{-y_{n}} y_{n}+e^{-y_{n}} y_{n}-e^{-\bar{y}} y_{n}\right) \\
& +\frac{c h}{\left(d+h y_{n}\right)(d+h \bar{y})} \\
& \times\left(e^{-x_{n}} \bar{y}-e^{-x_{n}} y_{n}+e^{-x_{n}} y_{n}-e^{-\bar{y}} y_{n}\right) \\
& -\frac{a h}{\left(d+h y_{n}\right)(d+h \bar{y})}\left(y_{n}-\bar{y}\right) \\
& =\frac{-b}{e^{y_{n}+\bar{y}}(d+h \bar{y})}\left(e^{y_{n}}-e^{\bar{y}}\right)
\end{aligned}
$$

$$
\begin{aligned}
& -\frac{c}{e^{x_{n}+\bar{x}}(d+h \bar{y})}\left(e^{x_{n}}-e^{\bar{x}}\right) \\
& -h \cdot \frac{a+b e^{-y_{n}}+c e^{-x_{n}}}{\left(d+h y_{n}\right)(d+h \bar{y})}\left(y_{n}-\bar{y}\right) \\
= & \frac{-b}{e^{y_{n}}(d+h \bar{y})}\left(e^{y_{n}-\bar{y}}-1\right) \\
& -\frac{c}{e^{x_{n}}(d+h \bar{y})}\left(e^{x_{n}-\bar{x}}-1\right) \\
& -h \cdot \frac{a+b e^{-y_{n}}+c e^{-x_{n}}}{\left(d+h y_{n}\right)(d+h \bar{y})}\left(y_{n}-\bar{y}\right) \\
= & \frac{-b}{e^{y_{n}}(d+h \bar{y})}\left[\left(y_{n}-\bar{y}\right)+\mathcal{O}_{2}\left(\left(y_{n}-\bar{y}\right)^{2}\right)\right] \\
& -\frac{c}{e^{x_{n}}(d+h \bar{y})}\left[\left(x_{n}-\bar{x}\right)+\mathcal{O}_{1}\left(\left(x_{n}-\bar{x}\right)^{2}\right)\right] \\
& -h \cdot \frac{a+b e^{-y_{n}}+c e^{-x_{n}}}{\left(d+h y_{n}\right)(d+h \bar{y})}\left(y_{n}-\bar{y}\right) \\
= & \frac{-c}{e^{x_{n}}(d+h \bar{y})}\left(x_{n}-\bar{x}\right) \\
& +\frac{-b e^{-y_{n}}\left(d+h y_{n}\right)-h\left(a+b e^{-y_{n}}+c e^{-x_{n}}\right)}{\left(d+h y_{n}\right)(d+h \bar{y})} \\
& \times\left(y_{n}-\bar{y}\right) \\
& +\mathcal{O}_{1}\left(\left(x_{n}-\bar{x}\right)^{2}\right)+\mathcal{O}_{2}\left(\left(y_{n}-\bar{y}\right)^{2}\right) . \\
& -h(d 8)
\end{aligned}
$$

By calculating similarly, we get

$$
\begin{aligned}
& y_{n+1}-\bar{y} \\
&=\frac{-b e^{-x_{n}}\left(d+h x_{n}\right)-h\left(a+b e^{-x_{n}}+c e^{-y_{n}}\right)}{\left(d+h x_{n}\right)(d+h \bar{x})}\left(x_{n}-\bar{x}\right) \\
&+\frac{-c}{e^{y_{n}}(d+h \bar{x})}\left(y_{n}-\bar{y}\right) \\
&+\mathcal{O}_{3}\left(\left(x_{n}-\bar{x}\right)^{2}\right)+\mathcal{O}_{4}\left(\left(y_{n}-\bar{y}\right)^{2}\right) .
\end{aligned}
$$

From (28) and (29) we have

$$
\begin{aligned}
x_{n+1}-\bar{x} \approx & \frac{-c}{e^{x_{n}}(d+h \bar{y})}\left(x_{n}-\bar{x}\right) \\
& +\frac{-b e^{-y_{n}}\left(d+h y_{n}\right)-h\left(a+b e^{-y_{n}}+c e^{-x_{n}}\right)}{\left(d+h y_{n}\right)(d+h \bar{y})} \\
& \times\left(y_{n}-\bar{y}\right),
\end{aligned}
$$




$$
\begin{aligned}
y_{n+1}-\bar{y} \approx & \frac{-b e^{-x_{n}}\left(d+h x_{n}\right)-h\left(a+b e^{-x_{n}}+c e^{-y_{n}}\right)}{\left(d+h x_{n}\right)(d+h \bar{x})} \\
& \times\left(x_{n}-\bar{x}\right)+\frac{-c}{e^{y_{n}}(d+h \bar{x})}\left(y_{n}-\bar{y}\right) .
\end{aligned}
$$

Set

$$
e_{n}^{1}=x_{n}-\bar{x}, \quad e_{n}^{2}=y_{n}-\bar{y}
$$

Then system (30) can be represented as

$$
\begin{aligned}
& e_{n+1}^{1} \approx a_{n} e_{n}^{1}+b_{n} e_{n}^{2}, \\
& e_{n+1}^{2} \approx c_{n} e_{n}^{1}+d_{n} e_{n}^{2},
\end{aligned}
$$

where

$$
\begin{gathered}
a_{n}=\frac{-c}{e^{x_{n}}(d+h \bar{y})}, \\
b_{n}=\frac{-b e^{-y_{n}}\left(d+h y_{n}\right)-h\left(a+b e^{-y_{n}}+c e^{-x_{n}}\right)}{\left(d+h y_{n}\right)(d+h \bar{y})}, \\
c_{n}=\frac{-b e^{-x_{n}}\left(d+h x_{n}\right)-h\left(a+b e^{-x_{n}}+c e^{-y_{n}}\right)}{\left(d+h x_{n}\right)(d+h \bar{x})}, \\
d_{n}=\frac{-c}{e^{y_{n}}(d+h \bar{x})} .
\end{gathered}
$$

$$
A=\left(\begin{array}{c}
\frac{-c e^{-\bar{x}}}{d+h \bar{x}} \\
\frac{-b e^{-\bar{x}}(d+h \bar{x})-h \cdot\left[a+(b+c) e^{-\bar{x}}\right]}{(d+h \bar{x})^{2}}
\end{array}\right.
$$$$
B(n)=\left(\begin{array}{ll}
\alpha_{n} & \beta_{n} \\
\delta_{n} & \gamma_{n}
\end{array}\right), \quad\|B(n)\| \longrightarrow 0 \quad \text { as } n \longrightarrow \infty .
$$

that is

where

Taking the limits of $a_{n}, b_{n}, c_{n}$, and $d_{n}$ as $n \rightarrow \infty$, we obtain

$$
\begin{gathered}
\lim _{n \rightarrow \infty} a_{n}=\frac{-c}{e^{\bar{x}}(d+h \bar{x})}, \\
\lim _{n \rightarrow \infty} b_{n}=\frac{-b e^{-\bar{x}}(d+h \bar{x})-h\left[a+(b+c) e^{-\bar{x}}\right]}{(d+h \bar{x})^{2}}, \\
\lim _{n \rightarrow \infty} c_{n}=\frac{-b e^{-\bar{x}}(d+h \bar{x})-h\left[a+(b+c) e^{-\bar{x}}\right]}{(d+h \bar{x})^{2}}, \\
\lim _{n \rightarrow \infty} d_{n}=\frac{-c}{e^{\bar{x}}(d+h \bar{x})} ;
\end{gathered}
$$

$$
\begin{gathered}
a_{n}=\frac{-c}{e^{\bar{x}}(d+h \bar{x})}+\alpha_{n}, \\
b_{n}=\frac{-b e^{-\bar{x}}(d+h \bar{x})-h\left[a+(b+c) e^{-\bar{x}}\right]}{(d+h \bar{x})^{2}}+\beta_{n}, \\
c_{n}=\frac{-b e^{-\bar{x}}(d+h \bar{x})-h\left[a+(b+c) e^{-\bar{x}}\right]}{(d+h \bar{x})^{2}}+\gamma_{n}, \\
d_{n}=\frac{-c}{e^{\bar{x}}(d+h \bar{x})}+\delta_{n},
\end{gathered}
$$

where $\alpha_{n} \rightarrow 0, \beta_{n} \rightarrow 0, \gamma_{n} \rightarrow 0$, and $\delta_{n} \rightarrow 0$ as $n \rightarrow \infty$

Now, we have system of the form (18):

$$
\mathbf{e}_{n+1}=(A+B(n)) \mathbf{e}_{n},
$$

$$
\left.\frac{-b e^{-\bar{x}}(d+h \bar{x})-h \cdot\left[a+(b+c) e^{-\bar{x}}\right]}{(d+h \bar{x})^{2}}\right),
$$

Thus, the limiting system of error terms can be written as

$$
\left(\begin{array}{l}
e_{n+1}^{1} \\
e_{n+1}^{2}
\end{array}\right)=A\left(\begin{array}{l}
e_{n}^{1} \\
e_{n}^{2}
\end{array}\right)
$$

The system is exactly linearized system of (2) evaluated at the equilibrium $E=(\bar{x}, \bar{y})=(\bar{x}, \bar{x})$. Then Theorems 4 and 5 imply the result.

\section{Conflict of Interests}

The authors declare that there is no conflict of interests regarding the publication of this paper.

\section{References}

[1] I. Ozturk, F. Bozkurt, and S. Ozen, "On the difference equation $y_{n+1}=\left(\alpha+B e^{-y n}\right) /\left(\gamma+y_{n-1}\right), "$ Applied Mathematics and Computation, vol. 181, no. 2, pp. 1387-1393, 2006.

[2] D. C. Zhang and B. Shi, "Oscillation and global asymptotic stability in a discrete epidemic model," Journal of Mathematical Analysis and Applications, vol. 278, no. 1, pp. 194-202, 2003. 
[3] E. El-Metwally, E. A. Grove, G. Ladas, R. Levins, and M. Radin, "On the difference equation, $x_{n}+1=\left(a+\beta_{x n}-1\right) e^{-x_{n}}$," Nonlinear Analysis: Theory, Methods \& Applications, vol. 47, no. 7, pp. 4623-4634, 2001.

[4] G. Papaschinopoulos, M. A. Radin, and C. J. Schinas, "On the system of two difference equations of exponential form: $x_{n+1}=$ $a+b x_{n-1} e^{-y_{n}}, y_{n+1}=c+d y_{n-1} e^{x_{n}}$, Mathematical and Computer Modelling, vol. 54, no. 11-12, pp. 2969-2977, 2011.

[5] G. Stefanidou, G. Papaschinopoulos, and C. J. Schinas, "On a system of two exponential type difference equations," Communications on Applied Nonlinear Analysis, vol. 17, no. 2, pp. 1-13, 2010.

[6] S. Stević, "On a discrete epidemic model," Discrete Dynamics in Nature and Society, vol. 2007, Article ID 87519, 10 pages, 2007.

[7] E. A. Grove and G. Ladas, Periodicities in Nonlinear Difference Equations, Chapman \& Hall/CRC, 2005.

[8] R. P. Agarwal, Difference Equations and Inequalities, Marcel Dekker, New York, NY, USA, 2nd edition, 2000.

[9] V. L. Kocic and G. Ladas, Global Behavior of Nonlinear Difference Equations of Higher Order with Applications, Kluwer Academic, Dordrecht, The Netherlands, 1993.

[10] M. R. S. Kulenović and G. Ladas, Dynamics of Second Order Rational Difference Equations with Open Problems and Conjectures, Chapman and Hall/CRC, Boca Raton, Fla, USA, 2001.

[11] D. Burgić and Z. Nurkanović, "An example of a globally asymptotically stable anti-monotonic system of rational difference equations in the plane," Sarajevo Journal of Mathematics, vol. 5, no. 18, pp. 235-245, 2009.

[12] M. R. S. Kulenović and M. Nurkanović, "Asymptotic behavior of a competitive system of linear fractional difference equations," Advances in Difference Equations, vol. 2006, Article ID 019756, 2006.

[13] M. R. S. Kulenović and Z. Nurkanović, "The rate of convergence of solution of a three dimensional linear fractional systems of difference equations," Zbornik radova PMF Tuzla-Svezak Matematika, vol. 2, pp. 1-6, 2005.

[14] M. Pituk, "More on Poincare's and Peron's theorems for difference equations," Journal of Difference Equations and Applications, vol. 8, pp. 201-216, 2002. 


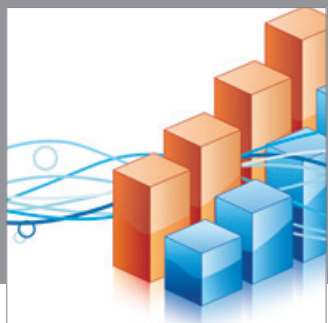

Advances in

Operations Research

mansans

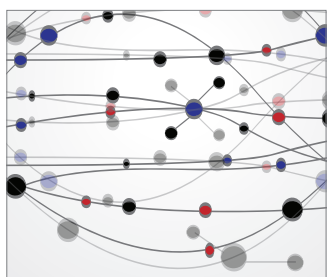

The Scientific World Journal
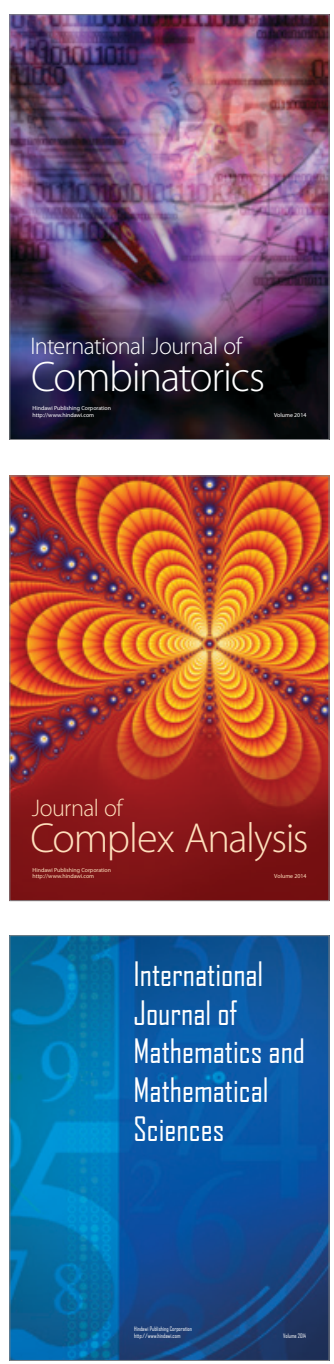
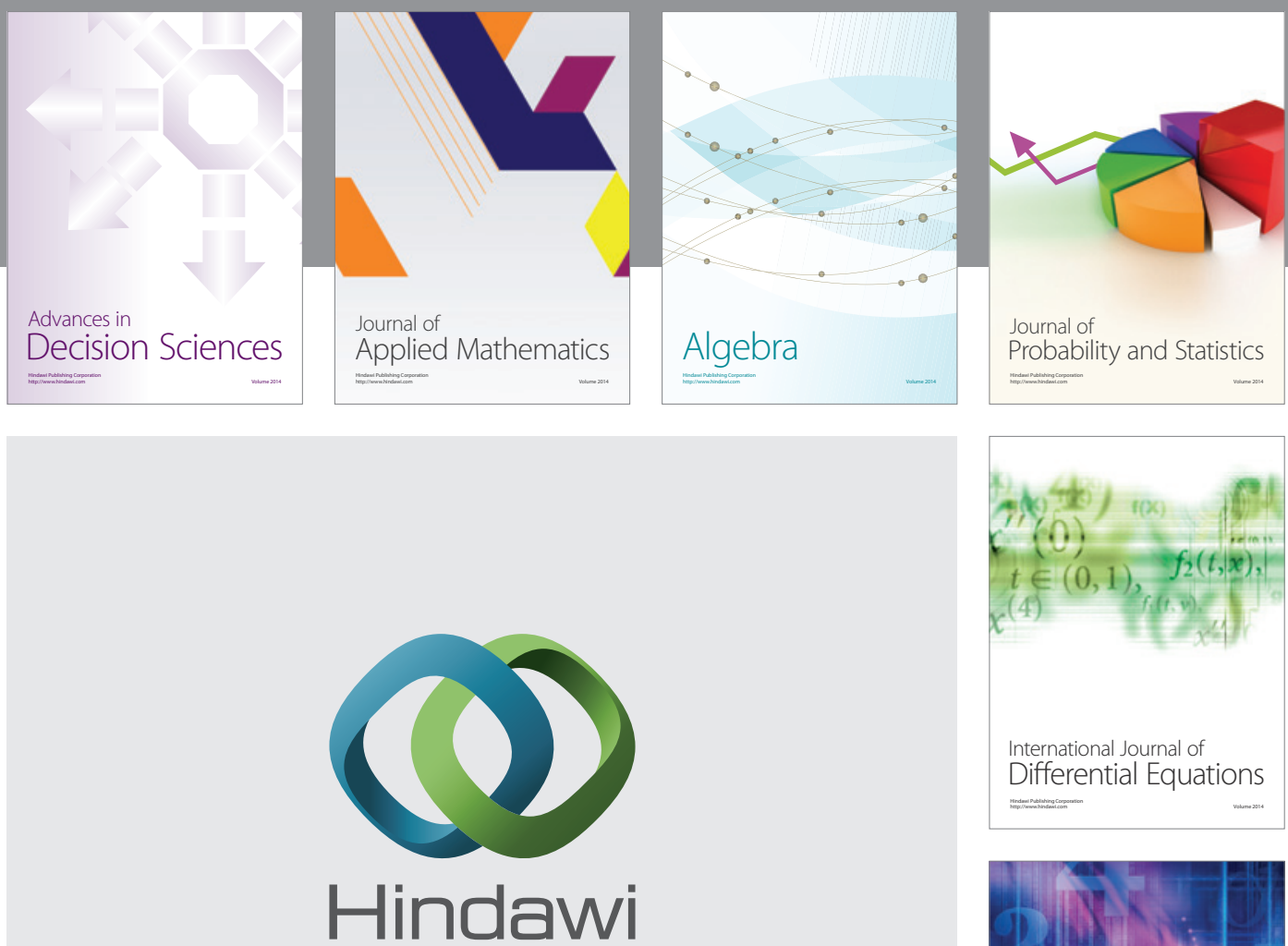

Submit your manuscripts at http://www.hindawi.com
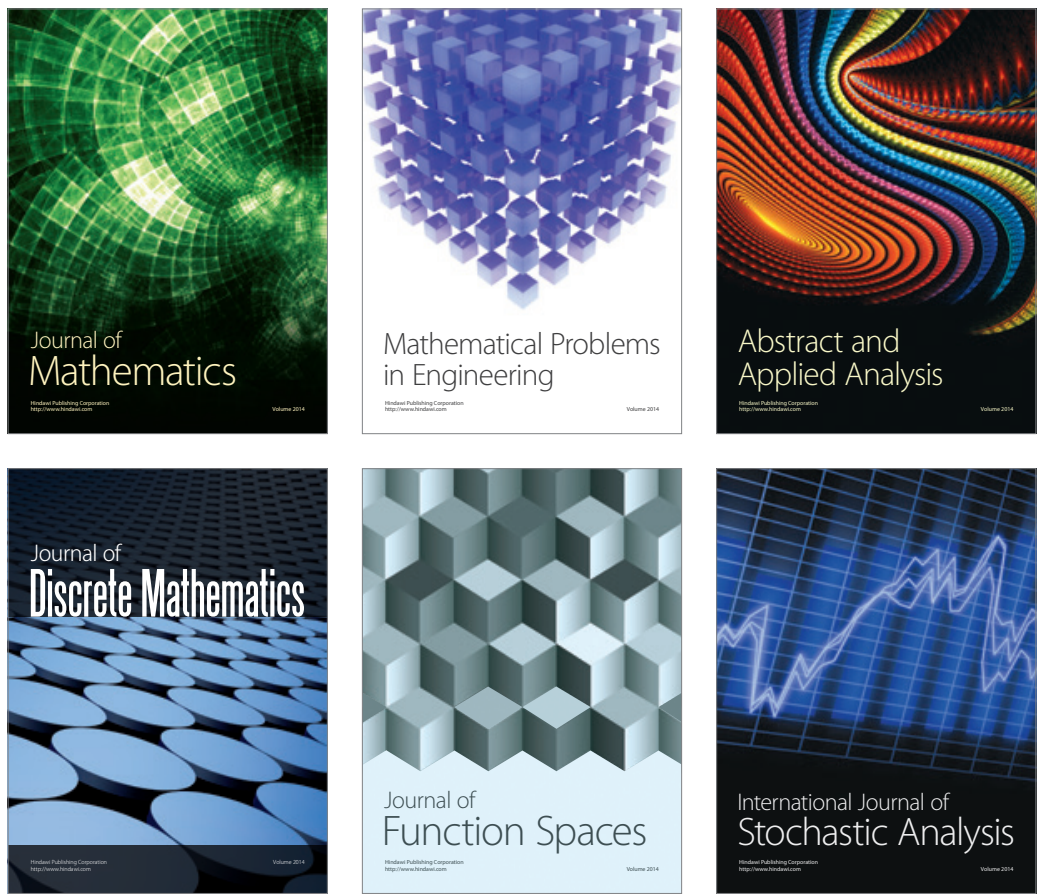

Journal of

Function Spaces

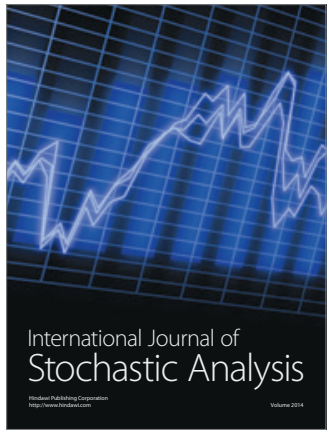

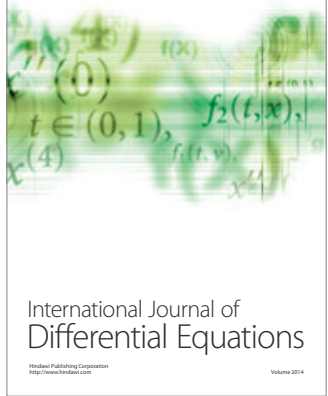
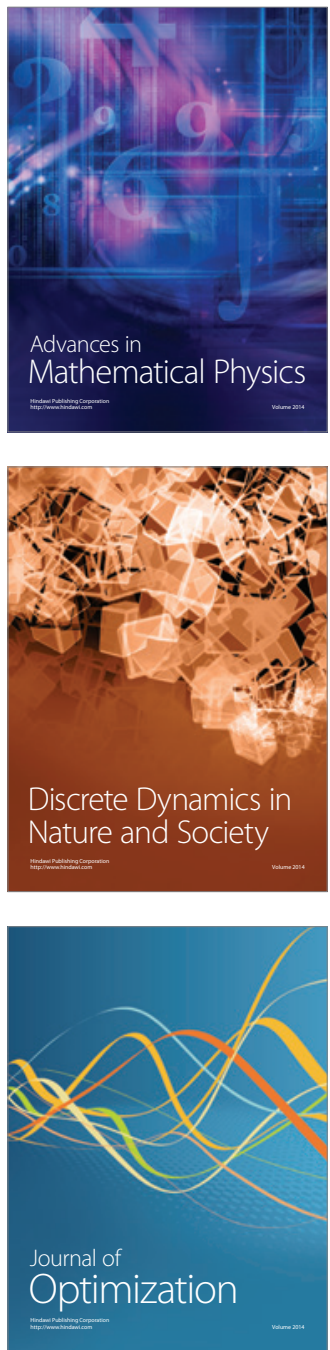